\title{
Partial state and input observability recovering by additional sensor implementation: \\ A graph-theoretic approach
}

\author{
T. Boukhobza \\ Centre de Recherche en Automatique de Nancy (CRAN), Nancy-University, CNRS UMR 7039 \\ email: taha.boukhobza@cran.uhp-nancy.fr
}

\begin{abstract}
This paper deals with the problem of additional sensor location in order to recover the observability of any given part of the state and unknown input for structured linear systems. The proposed method is based on a graph-theoretic approach and assumes only the knowledge of the system's structure. We first provide graphical necessary and sufficient conditions for the generic observability of any given part of the state and input. Then, we study the number and location of additional sensors in order to satisfy the latter conditions. On the one hand, we provide necessary requirements to be satisfied by these additional sensors. on the other hand, we give other sufficient simple conditions allowing to add a number, which is is guaranteed to be minimal, of sensors to ensure the observability of any given part of the state and unknown input.
\end{abstract}

Keywords: Partial state and input generic observability, sensor location, structured linear systems, graph theory.

\section{Introduction}

The problem of estimating a part of the state and the unknown input is of great interest mainly in control law synthesis, fault detection and isolation, fault tolerant control, supervision and so on. Indeed, in many applications, the estimation of only a part of the state and the unknown input of the system is necessary. This may be very useful, for example, when we want to preserve the state observability in the context of a system submitted to faults or in the case of network distributed systems submitted to network cuts or failures. In this respect, 
many works (Koenig, 2005; Chu, 2000; Chu and Mehrmann, 1999; Hou et al., 1999; Hou and Müller, 1992; Trinh and Ha, 2000; Tsui, 1996; Kudva et al., 1980) are focused on the design of full or reduced state observers for linear systems with unknown inputs. More recently, for variable time-delay uncertain systems with unknown inputs, the authors of (Gao and Wang, 2003; Darouach, 2005; Lin et al., 2007) propose observers to reconstruct a given part of the state. The proposed method is based on Linear Matrix Inequalities and Equalities (LMI and LME) and is obviously very well adapted for network distributed systems as it take into account variable delays.

A preliminary step to the observer design is the analysis of the state and input observability. Such analysis has been addressed in many studies which provide conditions on the state and/or input observability (Basile and Marro, 1973; Hou and Müller, 1999; Hou and Patton, 1998). Among the most important works dealing with the state reconstructibility, we can cite the approach developed in (Hautus, 1983) where the author gives the definitions of strong detectability and strong observability and the conditions for existence of observers that estimate a functional of the state and unknown inputs. When these conditions are not ensured, the only way to recover the observability, is to add sensors. Many studies reviewed in (van de Wal and de Jager, 2001) deal with the selection and sensor placement, which almost all use an optimisation criteria related to the observability Gramian, sensitivity functions .... To apply classical algebraic and geometric tools to address the sensors addition issue, the exact knowledge of the state space matrices characterizing the system's model is required. However, in many modeling problems, these matrices have a number of fixed zero entries determined by the physical laws while the remaining entries are not precisely known, particularly in the case of large systems or during a conception stage. This is why, to analyse these systems, in spite of the poor knowledge we have on them, the idea is that we only keep the zero/non-zero entries in the state space matrices. Thus, we consider models where the fixed zeros are conserved while the non-zero entries are replaced by free parameters.

There is a huge amount of interesting works in the literature using this kind of models called structured models. Many of these works are related to the graph-theoretic approach and aim to 
analyse some system properties such as controllability, observability or the solvability of several classical control problems including disturbance rejection, input-output decoupling, ... (Dion et al., 2003). It results from these works that the graph-theoretic approach provides simple and elegant solutions. Such approach has been also used for the sensor location studies. In this way, (Maquin et al., 1994; Meyer et al., 1994) tackle the problem of additional sensor location starting from some static observability equations which summarize all the available information data on the system. In (Commault et al., 2005), the authors address the problem of additional sensors to recover the state observability of linear structured systems. The suggested approach allows to obtain the minimal number of required additional sensors and the conditions they must satisfy in order to recover the state observability. Since our work is related to an observability problem, it is closer to the latter reference with the originality that we consider systems with exogenous unknown input and we try to recover both the state and input observability without any restriction on the input dynamics.

More precisely, in this paper, using a graph-theoretic approach, we study the additional sensor location in order to recover the strong observability of a desired part of the state and the input. At this aim, in a first stage, we provide the graphical necessary and sufficient conditions for the observability of any given part of the state and input components. Then, we study the number and the location of the additional sensors which allow to satisfy the latter observability conditions. In other words, the main contribution of the paper consists in three results. The first one lies in the graphical conditions for the strong observability of a state or an input component. Secondly, we provide the necessary conditions which must be satisfied by the additional sensors to ensure the strong observability of the given set of state and input components. Finally, we enounce some sufficient conditions, on the form of graphical equations, which ensure that a sensor configuration allows to recover the strong observability of the given set of state and input components. These conditions do not allow to provide the minimal number or an exhaustive list of all the possible sensors placements which solve the considered observability problem. Nevertheless, they allow to check quite easily if a given location is acceptable and they can lead to a simple procedure for the 
sensor placement during a system conception stage. Moreover, the proposed results are not trivial even if they are quite simple because based on searching paths and matchings in a digraph using well-known combinatorial algorithms. This makes our method particularly simple and intuitive and so adapted to large scale systems.

The paper is organised as follows: after Section 2, which is devoted to the problem formulation, a digraph representation of structured systems is given in Section 3. The main results are enounced in Section 4. An example illustrates the proposed sensor location strategy. Finally, some concluding remarks are made.

\section{Problem statement}

In this paper, we treat numerically non-specified systems on the form:

$$
\Sigma_{\Lambda}:\left\{\begin{array}{l}
\dot{x}(t)=A^{\lambda} x(t)+B^{\lambda} u(t) \\
y(t)=C^{\lambda} x(t)+D^{\lambda} u(t)
\end{array}\right.
$$

where $x \in \mathbb{R}^{n}, u \in \mathbb{R}^{q}$ and $y \in \mathbb{R}^{p}$ are respectively the state vector, the unknown input vector and the output vector. $A^{\lambda}, B^{\lambda}, C^{\lambda}$ and $D^{\lambda}$ represent matrices which elements are either fixed to zero or assumed to be nonzero free parameters noted $\lambda_{i}$. These parameters forms a vector $\Lambda=\left(\lambda_{1}, \ldots, \lambda_{h}\right)^{T} \in \mathbb{R}^{h}$.

If all parameters $\lambda_{i}$ are numerically fixed, we obtain a so-called admissible realization of structured system $\Sigma_{\Lambda}$. We say that a property is true generically (van der Woude, 2000) if it is true for almost all the realizations of structured system $\Sigma_{\Lambda}$. Here, "for almost all the realizations" is to be understood (Dion et al., 2003; van der Woude, 2000) as "for all parameter values $\left(\Lambda \in \mathbb{R}^{h}\right)$ except for those in some proper algebraic variety in the parameter space". The proper algebraic variety for which the property is not true is the zero set of some nontrivial polynomial with real coefficients in the $h$ system parameters $\lambda_{1}, \lambda_{2}, \ldots, \lambda_{h}$ or equivalently it is an algebraic variety which has Lebesgue measure zero.

In this paper, we study the problem of sensor implementation in order to guarantee the generic 
observability of a given part of the state and input for structured system $\Sigma_{\Lambda}$. Since we work with structured systems, the observation of a functional $L\left(x^{T}, u^{T}\right)^{T}$ has a meaning only if matrix $L$ is composed of euclidean vectors of $\mathbb{R}^{n+q}$ or in other words we analyse the additional sensor implementation in order to guarantee the strong observability of some state and input components. The state and input observability is related to both the strong observability and the left invertibility (Trentelman et al., 2001) properties. In other words, a system is state and input observable when all its state and input components can be expressed in function of the output components and their derivatives. The definition of the generic state and input observability is recalled hereafter:

Definition 1 Structured system $\Sigma_{\Lambda}$ is generically state and input observable iffor almost all realizations of $\Sigma_{\Lambda}$, for all initial states $x_{0}$ and for every input function $u(t), y(t)=0$ for $t \geq 0$ implies $x(t)=0$ for $t \geq 0$ and $u(t)=0$ for $t>0$.

Necessary and sufficient conditions for the state and input observability of structured system $\Sigma_{\Lambda}$ can be deduced from the ones provided in (Trentelman et al., 2001) or from the conditions of the right-hand side observability of a descriptor system given in Theorem 3 of (Hou and Müller, 1999). Thus, structured system $\Sigma_{\Lambda}$ is generically state and input observable iff

$$
\forall s \in \mathbb{C}, g \_r a n k(P(s))=n+g \_r a n k\left(\begin{array}{c}
B^{\lambda} \\
D^{\lambda}
\end{array}\right)=n+q
$$

where $P(s)=\left(\begin{array}{cc}A^{\lambda}-s I_{n} & B^{\lambda} \\ C^{\lambda} & D^{\lambda}\end{array}\right)$ is the matrix pencil of $\Sigma_{\Lambda}$ and $g \_r a n k(P(s))$ denotes the generic rank of matrix $P(s)$.

Hence, g_rank $(P(s))=r, \forall s \in \mathbb{C}$ means that for almost all parameter values $\Lambda \in \mathbb{R}^{h}$, $\operatorname{rank}(P(s))=r, \forall s \in \mathbb{C}$. Note that for each realization of system $\Sigma_{\Lambda}$, we can compute the $n$-rank of $P(s)$. This rank will have the same value for almost all parameter values $\Lambda \in \mathbb{R}^{h}$ (van der Woude, 2000). This so-called generic $n$-rank of $P(s)$ will be denoted by $g \_n$-rank $(P(s))$ which is different from $g \_r a n k(P(s))$ as the latter depends on $s$.

For the present study, we are interested in the generic strong observability of only a part of the state or the input. From Definition 1, the notion of strong observability of an input or a state component can be deduced as follows: 
Definition 2 Consider structured system $\Sigma_{\Lambda}$. State component $x_{i}$ (respectively input component $\left.u_{j}\right)$ is generically strongly observable if for all initial state $x_{0}$ and for every input function $u(t)$, $y(t)=0$ for $t \geq 0$ implies $x_{i}(t)=0, \forall t \geq 0$ (respectively $\left.u_{j}(t)=0, \forall t>0\right)$.

Roughly speaking, the generic strong observability of a state component $x_{i}$ (respectively an input component $u_{j}$ ) means that a change in $x_{i}(0)$ (respectively $u_{j}\left(0^{+}\right)$) is necessarily reflected in a change of measurements.

Starting from a system which does not satisfy condition (2), the objective of our study, which is based oh a graphic representation of the system, is to propose a strategy for additional sensor placement in order to recover the observability of any given set of state and input components. The proposed method must provide the number and the location of the necessary additional sensors as precisely as possible. At this aim, we must first provide the necessary and sufficient graphical condition for the strong observability of a state or input component. Then, we can address properly the sensor placement problem.

In this respect, the next section is dedicated to the definition of a graphical representation of linear structured systems and to some notations.

\section{Graphical representation of structured linear systems}

The directed graph or digraph $\mathcal{G}\left(\Sigma_{\Lambda}\right)$ associated to $\Sigma_{\Lambda}$ is constituted by a vertex set $\mathcal{V}$ and an edge set $\mathcal{E}$ i.e. $\mathcal{G}\left(\Sigma_{\Lambda}\right)=(\mathcal{V}, \mathcal{E})$. The vertices are associated to the state, the unknown input and the output components of $\Sigma_{\Lambda}$ whereas the edges represent links between these variables. More precisely, $\mathcal{V}=\mathbf{X} \cup \mathbf{Y} \cup \mathbf{U}$, where $\mathbf{X}=\left\{\mathbf{x}_{1}, \ldots, \mathbf{x}_{\mathbf{n}}\right\}$ is the set of state vertices, $\mathbf{Y}=\left\{\mathbf{y}_{\mathbf{1}}, \ldots, \mathbf{y}_{\mathbf{p}}\right\}$ is the set of output vertices and $\mathbf{U}=\left\{\mathbf{u}_{\mathbf{1}}, \ldots, \mathbf{u}_{\mathbf{q}}\right\}$ is the set of unknown input vertices. The edge set is $\mathcal{E}=A$-edges $\cup B$-edges $\cup C$-edges $\cup D$-edges, with $A$-edges $=\left\{\left(\mathbf{x}_{\mathbf{j}}, \mathbf{x}_{\mathbf{i}}\right) \mid A^{\lambda}(i, j) \neq 0\right\}, B$-edges $=\left\{\left(\mathbf{u}_{\mathbf{j}}, \mathbf{x}_{\mathbf{i}}\right) \mid B^{\lambda}(i, j) \neq 0\right\}, C$-edges $=$ $\left\{\left(\mathbf{x}_{\mathbf{j}}, \mathbf{y}_{\mathbf{i}}\right) \mid C^{\lambda}(i, j) \neq 0\right\}, D$-edges $=\left\{\left(\mathbf{u}_{\mathbf{j}}, \mathbf{y}_{\mathbf{i}}\right) \mid D^{\lambda}(i, j) \neq 0\right\}$, where $M^{\lambda}(i, j)$ is the $(i, j)$ th element of matrix $M^{\lambda}$ and $\left(\mathbf{v}_{\mathbf{1}}, \mathbf{v}_{\mathbf{2}}\right)$ denotes a directed edge from vertex $\mathbf{v}_{\mathbf{1}} \in \mathcal{V}$ to vertex $\mathbf{v}_{\mathbf{2}} \in \mathcal{V}$. 
Hereafter, we illustrate the proposed digraph representation with an example.

Example 1 To the system defined by the following matrices, we associate the digraph in Figure

\begin{tabular}{|c|c|c|c|c|c|c|c|c|c|c|c|c|c|}
\hline \multirow{4}{*}{1.} & $\lambda_{1}$ & 0 & 0 & 0 & 0 & 0 & 0 & 0 & 0 & 0 & 0 & 0 & 0 \\
\hline & $\lambda_{2}$ & 0 & 0 & 0 & 0 & 0 & 0 & 0 & 0 & 0 & 0 & 0 & $\lambda_{13}$ \\
\hline & 0 & $\lambda_{3}$ & 0 & $\lambda_{4}$ & 0 & 0 & 0 & 0 & 0 & 0 & $\lambda_{14}$ & $\lambda_{15}$ & 0 \\
\hline & 0 & 0 & $\lambda_{5}$ & 0 & 0 & 0 & 0 & 0 & 0 & 0 & 0 & 0 & 0 \\
\hline \multirow{6}{*}{$A^{\lambda}=$} & 0 & 0 & 0 & $\lambda_{6}$ & 0 & $\lambda_{7}$ & $\lambda_{8}$ & 0 & 0 & $R^{\lambda}-$ & 0 & 0 & 0 \\
\hline & 0 & 0 & $\lambda_{9}$ & $\lambda_{10}$ & 0 & 0 & 0 & 0 & 0 & 0 & 0 & 0 & 0 \\
\hline & 0 & 0 & 0 & 0 & 0 & 0 & 0 & 0 & 0 & 0 & 0 & $\lambda_{16}$ & 0 \\
\hline & 0 & & & 0 & 0 & & 0 & 0 & & & 0 & $\lambda_{17}$ & 0 \\
\hline & 0 & 0 & 0 & 0 & 0 & 0 & 0 & $\lambda_{12}$ & 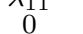 & 0 & 0 & 0 & $\begin{array}{l}0 \\
0\end{array}$ \\
\hline & 0 & $\begin{array}{ll}0 & 0\end{array}$ & 0 & $\lambda_{18}$ & 0 & 0 & 0 & 0 & 0 & & 0 & & \\
\hline \multirow{3}{*}{$C^{\lambda}=$} & 0 & 0 & 0 & $\lambda_{19}$ & 0 & 0 & 0 & $\lambda_{20}$ & 0 & $D^{\lambda}-$ & 0 & 0 & \\
\hline & 0 & 0 & 0 & 0 & 0 & $\lambda_{21}$ & 0 & 0 & $\lambda_{22}$ & I & 0 & 0 & - \\
\hline & & 0 & 0 & 0 & 0 & 0 & $\lambda_{23}$ & & 0 & & $\lambda_{24}$ & & \\
\hline
\end{tabular}

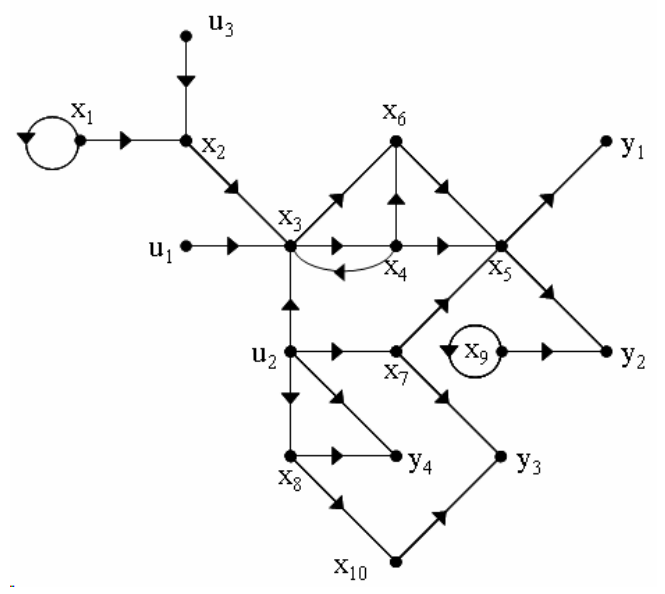

Figure 1: Digraph associated to system of Example 1

Let us now give some useful definitions and notations.

- Two edges $e_{1}=\left(\mathbf{v}_{\mathbf{1}}, \mathbf{v}_{\mathbf{1}}^{\prime}\right)$ and $e_{2}=\left(\mathbf{v}_{\mathbf{2}}, \mathbf{v}_{\mathbf{2}}^{\prime}\right)$ are $v$-disjoint if $\mathbf{v}_{\mathbf{1}} \neq \mathbf{v}_{\mathbf{2}}$ and $\mathbf{v}_{\mathbf{1}}^{\prime} \neq \mathbf{v}_{\mathbf{2}}^{\prime}$. Note that $e_{1}$ and $e_{2}$ can be $v$-disjoint even if $\mathbf{v}_{\mathbf{1}}^{\prime}=\mathbf{v}_{\mathbf{2}}$ or $\mathbf{v}_{\mathbf{1}}=\mathbf{v}_{\mathbf{2}}^{\prime}$.

Some edges are $v$-disjoint if they are mutually $v$-disjoint.

- We denote path $P$ containing vertices $\mathbf{v}_{\mathbf{r}_{0}}, \ldots, \mathbf{v}_{\mathbf{r}_{\mathbf{i}}}$ by $P=\mathbf{v}_{\mathbf{r}_{0}} \rightarrow \mathbf{v}_{\mathbf{r}_{1}} \rightarrow \ldots \rightarrow \mathbf{v}_{\mathbf{r}_{\mathbf{i}}}$, where $\left(\mathbf{v}_{\mathbf{r}_{\mathbf{j}}}, \mathbf{v}_{\mathbf{r}_{\mathbf{j}+\mathbf{1}}}\right) \in \mathcal{E}$ for $j=0,1, \ldots, i-1$. We say that $P$ covers $\mathbf{v}_{\mathbf{r}_{\mathbf{0}}}, \mathbf{v}_{\mathbf{r}_{\mathbf{1}}}, \ldots, \mathbf{v}_{\mathbf{r}_{\mathbf{i}}}$. A path is simple when every vertex occurs only once in this path.

- A cycle is a path of the form $\mathbf{v}_{\mathbf{r}_{0}} \rightarrow \mathbf{v}_{\mathbf{r}_{1}} \rightarrow \ldots \rightarrow \mathbf{v}_{\mathbf{r}_{\mathbf{i}}} \rightarrow \mathbf{v}_{\mathbf{r}_{0}}$, where all vertices $\mathbf{v}_{\mathbf{r}_{0}}, \mathbf{v}_{\mathbf{r}_{1}}, \ldots, \mathbf{v}_{\mathbf{r}_{\mathbf{i}}}$ are distinct.

- Some paths (resp. cycles) are disjoint if they have no common vertex. A set of disjoint cycles is called a cycle family. 
- Path $P$ is an $\mathbf{Y}$-topped path if its end vertex is an element of $\mathbf{Y}$. An $\mathbf{Y}$-topped path family consists of disjoint simple $\mathbf{Y}$-topped paths.

- The union of a Y-topped path family, and a cycle family is disjoint if they have no vertices in common. If such union contains path or a cycle which covers a vertex $\mathbf{v}$ it is said to cover $\mathbf{v}$.

- Let $\mathcal{V}_{1}$ and $\mathcal{V}_{2}$ denote two subsets of $\mathcal{V}$. The cardinality of $\mathcal{V}_{1}$ is noted $\operatorname{card}\left(\mathcal{V}_{1}\right)$. A path $P=\mathbf{v}_{\mathbf{r}_{\mathbf{0}}} \rightarrow \mathbf{v}_{\mathbf{r}_{1}} \rightarrow \ldots \rightarrow \mathbf{v}_{\mathbf{r}_{\mathbf{i}}}$ is said a $\mathcal{V}_{1}-\mathcal{V}_{2}$ path if $\mathbf{v}_{\mathbf{r}_{\mathbf{0}}} \in \mathcal{V}_{1}$ and $\mathbf{v}_{\mathbf{r}_{\mathbf{i}}} \in \mathcal{V}_{2}$. Moreover, if the only vertex of $P$ which belongs to $\mathcal{V}_{1}$ is $\mathbf{v}_{\mathbf{r}_{\mathbf{0}}}$ and the only vertex of $P$ which belongs to $\mathcal{V}_{2}$ is $\mathbf{v}_{\mathbf{r}_{\mathbf{i}}}, P$ is called a direct $\mathcal{V}_{1}-\mathcal{V}_{2}$ path.

- A set of $l$ disjoint $\mathcal{V}_{1}-\mathcal{V}_{2}$ paths is called a $\mathcal{V}_{1}-\mathcal{V}_{2}$ linking of size $l$. The linkings which consist of a maximal number of disjoint $\mathcal{V}_{1}-\mathcal{V}_{2}$ paths are called maximum $\mathcal{V}_{1}-\mathcal{V}_{2}$ linkings. We define by $\rho\left[\mathcal{V}_{1}, \mathcal{V}_{2}\right]$ the size of these maximum $\mathcal{V}_{1}-\mathcal{V}_{2}$ linkings.

- $\mu\left[\mathcal{V}_{1}, \mathcal{V}_{2}\right]$ is the minimal number of vertices covered by a maximum $\mathcal{V}_{1}-\mathcal{V}_{2}$ linking.

- $\theta\left(\mathcal{V}_{1}, \mathcal{V}_{2}\right)$ is the maximal number of $v$-disjoint edges which start in $\mathcal{V}_{1}$ and end in $\mathcal{V}_{2}$.

- The set of all essential vertices for the maximum $\mathcal{V}_{1}-\mathcal{V}_{2}$ linkings, noted $V_{\text {ess }}\left(\mathcal{V}_{1}, \mathcal{V}_{2}\right)$, is defined by $V_{\text {ess }}\left(\mathcal{V}_{1}, \mathcal{V}_{2}\right) \stackrel{\text { def }}{=}\left\{\mathbf{v} \in \mathcal{V} \mid \mathbf{v}\right.$ is included in every maximum $\mathcal{V}_{1}-\mathcal{V}_{2}$ linking $\}$.

- $\mathrm{S} \subseteq \mathcal{V}$ is a separator between sets $\mathcal{V}_{1}$ and $\mathcal{V}_{2}$, if every path from $\mathcal{V}_{1}$ to $\mathcal{V}_{2}$ contains at least one vertex in $\mathrm{S}$. We call minimum separators between $\mathcal{V}_{1}$ and $\mathcal{V}_{2}$ any separators having the smallest size. According to Menger's Theorem, the latter equals $\rho\left[\mathcal{V}_{1}, \mathcal{V}_{2}\right]$.

There is an uniquely determined minimum separator between $\mathcal{V}_{1}$ and $\mathcal{V}_{2}$ noted $\mathbf{S}_{*}\left(\mathcal{V}_{1}, \mathcal{V}_{2}\right)$, called minimum output separator (van der Woude, 2000) and which is the set of start vertices of all direct $V_{\text {ess }}\left(\mathcal{V}_{1}, \mathcal{V}_{2}\right)-\mathcal{V}_{2}$ paths, where $V_{\text {ess }}\left(\mathcal{V}_{1}, \mathcal{V}_{2}\right) \cap \mathcal{V}_{2}$ is considered, in the present definition, as input vertices. Obviously, $V_{\text {ess }}\left(\mathcal{V}_{1}, \mathcal{V}_{2}\right) \cap \mathcal{V}_{2} \subseteq \mathbf{S}_{*}\left(\mathcal{V}_{1}, \mathcal{V}_{2}\right)$

In Example 1, $\rho[\mathbf{U}, \mathbf{Y}]=2$ and $\theta(\mathbf{U}, \mathbf{Y})=1$. Moreover, $V_{\text {ess }}(\mathbf{U}, \mathbf{Y})=\left\{\mathbf{x}_{\mathbf{3}}, \mathbf{x}_{\mathbf{5}}, \mathbf{u}_{\mathbf{2}}\right\}$ and $\mathbf{S}_{*}(\mathbf{U}, \mathbf{Y})=\left\{\mathbf{x}_{\mathbf{5}}, \mathbf{u}_{\mathbf{2}}\right\}$.

We give now the following important definition:

Definition 3 For each vertex subset $\mathrm{V}$ such that $\mathrm{Y} \subseteq \mathrm{V} \subseteq \mathbf{X} \cup \mathbf{U} \cup \mathrm{Y}$, we associate the following vertex subsets: 
- $\overline{\mathbf{X}}(\mathbf{V})=\mathbf{X} \backslash(\mathbf{V} \cap \mathbf{X})$;

- $\overline{\mathbf{U}}(\mathbf{V}) \subseteq \mathbf{U}$ such that $\operatorname{card}(\overline{\mathbf{U}}(\mathbf{V}))=\rho[\mathbf{U}, \mathbf{V}]=\rho[\overline{\mathbf{U}}(\mathbf{V}), \mathbf{V}]$ and $\mu[\overline{\mathbf{U}}(\mathbf{V}), \mathbf{V}]=\mu[\mathbf{U}, \mathbf{V}]$.

Note that $\overline{\mathbf{U}}(\mathbf{V})$ always exists but is not necessarily unique.

- $\mathbf{X}_{\mathbf{1}}(\mathbf{V}) \stackrel{\text { def }}{=}\left\{\mathbf{x}_{\mathbf{i}} \in \overline{\mathbf{X}}(\mathbf{V}) \mid \rho\left[\mathbf{U} \cup\left\{\mathbf{x}_{\mathbf{i}}\right\}, \mathbf{V}\right]>\rho[\mathbf{U}, \mathbf{V}]\right\}$;

- $\boldsymbol{\Upsilon}_{\mathbf{0}}(\mathbf{V}) \stackrel{\text { def }}{=}\left\{\mathbf{v}_{\mathbf{i}} \in \mathbf{V} \mid \rho[\mathbf{U}, \mathbf{V}]>\rho\left[\mathbf{U}, \mathbf{V} \backslash\left\{\mathbf{v}_{\mathbf{i}}\right\}\right]\right\}=\mathbf{V} \cap V_{\text {ess }}(\mathbf{U}, \mathbf{V})$;

- $\Upsilon_{\mathbf{1}}(\mathbf{V}) \stackrel{\text { def }}{=} \mathrm{V} \backslash \Upsilon_{\mathbf{0}}(\mathbf{V})$;

- $\mathbf{U}_{\mathbf{0}}(\mathbf{V}) \stackrel{\text { def }}{=}\left\{\mathbf{u}_{\mathbf{i}} \in \overline{\mathbf{U}}(\mathbf{V}) \mid \theta\left(\left\{\mathbf{u}_{\mathbf{i}}\right\}, \mathbf{X}_{\mathbf{1}}(\mathbf{V}) \cup \boldsymbol{\Upsilon}_{\mathbf{1}}(\mathbf{V})\right)=0\right\}$;

- $\mathbf{U}_{\mathbf{1}}(\mathbf{V}) \stackrel{\text { def }}{=} \overline{\mathbf{U}}(\mathbf{V}) \backslash \mathbf{U}_{\mathbf{0}}(\mathbf{V})$;

- $\mathbf{S}_{*}(\mathbf{V}) \stackrel{\text { def }}{=} \mathbf{S}_{*}\left(\mathbf{U}_{\mathbf{0}}(\mathbf{V}), \mathbf{V}\right)$;

- $\mathbf{X}_{\mathbf{s}}(\mathbf{V}) \stackrel{\text { def }}{=} \mathbf{S}_{*}(\mathbf{V}) \cap \overline{\mathbf{X}}(\mathbf{V})$.

- $\mathbf{X}_{\mathbf{0}}(\mathbf{V}) \stackrel{\text { def }}{=} \overline{\mathbf{X}}(\mathbf{V}) \backslash\left(\mathbf{X}_{\mathbf{1}}(\mathbf{V}) \cup \mathbf{X}_{\mathbf{s}}(\mathbf{V})\right)$.

- $\beta_{1}(\mathbf{V})$ is the maximal number of vertices included in $\mathbf{X}_{\mathbf{1}}(\mathbf{V}) \cup \mathbf{X}_{\mathbf{s}}(\mathbf{V}) \cup \mathbf{U}_{\mathbf{1}}(\mathbf{V})$ covered by a disjoint union of

- $a \mathbf{X}_{\mathbf{s}}(\mathbf{V}) \cup \mathbf{U}_{\mathbf{1}}(\mathbf{V})-\Upsilon_{\mathbf{1}}(\mathbf{V})$ linking of size $\rho\left[\mathbf{X}_{\mathbf{s}}(\mathbf{V}) \cup \mathbf{U}_{\mathbf{1}}(\mathbf{V}), \Upsilon_{\mathbf{1}}(\mathbf{V})\right]$,

- a $\Upsilon_{1}(\mathbf{V})$-topped path family and

- a cycle family covering only elements of $\mathbf{X}_{\mathbf{1}}(\mathbf{V})$.

- $\beta_{0}(\mathbf{V}) \stackrel{\text { def }}{=} \mu\left[\mathbf{U}_{\mathbf{0}}(\mathbf{V}), \mathbf{S}_{*}(\mathbf{V})\right]-\rho\left[\mathbf{U}_{\mathbf{0}}(\mathbf{V}), \mathbf{S}_{*}(\mathbf{V})\right]$;

- $\beta(\mathbf{V}) \stackrel{\text { def }}{=} \beta_{1}(\mathbf{V})+\beta_{0}(\mathbf{V})+\operatorname{card}(\mathbf{V} \backslash \mathbf{Y})$.

To illustrate the previous definition, in the case of the system described in Example 1, we have, $\overline{\mathbf{X}}(\mathbf{Y})=\mathbf{X}, \overline{\mathbf{U}}(\mathbf{Y})=\left\{\mathbf{u}_{1}, \mathbf{u}_{2}\right\}, \mathbf{X}_{1}(\mathbf{Y})=\left\{\mathbf{x}_{7}, \mathbf{x}_{8}, \mathbf{x}_{9}, \mathbf{x}_{10}\right\}, \Upsilon_{\mathbf{0}}(\mathbf{Y})=\emptyset$, $\mathbf{\Upsilon}_{\mathbf{1}}(\mathbf{Y})=\mathbf{Y}, \mathbf{U}_{\mathbf{0}}(\mathbf{Y})=\left\{\mathbf{u}_{\mathbf{1}}\right\}, \mathbf{U}_{\mathbf{1}}(\mathbf{Y})=\left\{\mathbf{u}_{\mathbf{2}}\right\}, \mathbf{S}_{*}(\mathbf{Y})=\left\{\mathbf{x}_{\mathbf{5}}\right\}=\mathbf{X}_{\mathbf{s}}(\mathbf{Y}), \mathbf{X}_{\mathbf{0}}(\mathbf{Y})=$ $\left\{\mathrm{x}_{\mathbf{1}}, \mathrm{x}_{\mathbf{2}}, \mathrm{x}_{\mathbf{3}}, \mathrm{x}_{\mathbf{4}}, \mathrm{x}_{\mathbf{6}}\right\}, \beta_{0}(\mathbf{Y})=4-1$. Moreover, since $\mathrm{x}_{\mathbf{9}} \rightarrow \mathrm{x}_{\mathbf{9}}$ is the only cycle in $\mathbf{X}_{\mathbf{1}}(\mathbf{Y})$ and $\rho\left[\mathbf{X}_{\mathbf{s}}(\mathbf{Y}) \cup \mathbf{U}_{\mathbf{1}}(\mathbf{Y}), \mathbf{\Upsilon}_{\mathbf{1}}(\mathbf{Y})\right]=2$, the maximal number of state and input vertices covered by the disjoint union of two $\mathbf{X}_{\mathbf{s}}(\mathbf{Y}) \cup \mathbf{U}_{1}(\mathbf{Y})-\Upsilon_{1}(\mathbf{Y})$ paths and a $\Upsilon_{1}(\mathbf{Y})$-topped path family which does not cover $\mathrm{x}_{9}$ is $4: \mathbf{u}_{2} \rightarrow \mathrm{x}_{8} \rightarrow \mathrm{x}_{10} \rightarrow \mathrm{y}_{3}$ and $\mathrm{x}_{5} \rightarrow \mathrm{y}_{1}$. So, $\beta_{1}(\mathbf{Y})=5$ and then $\beta(\mathbf{Y})=5+(4-1)+0=8$.

In (Boukhobza et al., 2007), the subdivision of the system described above is introduced. Mainly, 
it satisfies $V_{\text {ess }}(\overline{\mathbf{U}}(\mathbf{V}), \mathbf{V})=V_{\text {ess }}\left(\mathbf{U}_{\mathbf{0}}(\mathbf{V}), \mathbf{V}\right) \cup \mathbf{U}_{\mathbf{1}}(\mathbf{V}), \theta\left(\mathbf{X}_{\mathbf{s}}(\mathbf{V}), \mathbf{X}_{\mathbf{1}}(\mathbf{V}) \cup \mathbf{\Upsilon}_{\mathbf{1}}(\mathbf{V})\right)=$ $\operatorname{card}\left(\mathbf{X}_{\mathbf{s}}(\mathbf{V})\right), \mathbf{S}_{*}(\overline{\mathbf{U}}(\mathbf{V}), \mathbf{V})=\mathbf{X}_{\mathbf{s}}(\mathbf{V}) \cup \boldsymbol{\Upsilon}_{\mathbf{0}}(\mathbf{V}) \cup \mathbf{U}_{\mathbf{1}}(\mathbf{V})$ and $\theta\left(\mathbf{X}_{\mathbf{0}}(\mathbf{V}) \cup \boldsymbol{\Upsilon}_{\mathbf{0}}(\mathbf{V}), \mathbf{X}_{\mathbf{1}}(\mathbf{V}) \cup\right.$ $\left.\Upsilon_{\mathbf{1}}(\mathbf{V})\right)=0$.

Consider a vertex set $\mathrm{V}$ such that $\mathrm{Y} \subseteq \mathrm{V} \subseteq \mathrm{X} \cup \mathrm{U} \cup \mathrm{Y}$ and assume that some sensors measuring the components associated to $\mathrm{V} \backslash \mathrm{Y}$ have been added to system $\Sigma_{\Lambda}$. The latter equalities allow us to study the input and state observability of such augmented system $\Sigma_{\Lambda}$ by considering independently two systems:

- a square system denoted $\Sigma_{0}$, defined by input $\mathbf{U}_{\mathbf{0}}(\mathbf{V})$, state $\mathbf{X}_{\mathbf{0}}(\mathbf{V})$ and output $\mathbf{X}_{\mathbf{s}}(\mathbf{V}) \cup \Upsilon_{\mathbf{0}}(\mathbf{V})$, - a system denoted $\Sigma_{1}$, defined by input $\mathbf{U}_{\mathbf{1}}(\mathbf{V}) \cup \mathbf{X}_{\mathbf{s}}(\mathbf{V})$, state $\mathbf{X}_{\mathbf{1}}(\mathbf{V})$ and output $\Upsilon_{\mathbf{1}}(\mathbf{V})$.

The particularity of the proposed subdivision is that the input and state observability of $\Sigma_{\Lambda}$ can be done by studying separately $\Sigma_{0}$ and $\Sigma_{1}$ (Boukhobza et al., 2007). In fact, the subdivision corresponds to a particular decomposition of the matrix pencil of the system similar to the one suggested in (van der Woude et al., 2003) but it is here explicitly defined. Moreover, it is well adapted to the application of the two main theorems of (van der Woude, 2000) characterizing the generic rank of the pencil matrix of a structured system in the two particular cases of a square system and a particular rectangular system.

\section{Main results}

Before addressing the sensor placement problem, in a first subsection, we give the necessary and sufficient condition which ensures the generic strong observability of state component $x_{i}$ or input component $u_{i}$. These conditions are deduced conjointly from (Boukhobza et al., 2007; van der Woude et al., 2003) and use function $\beta$ defined at the end of Section 3.

\subsection{Partial strong observability analysis}

The first obvious necessary condition to the strong observability of a state component $x_{i}$ (respectively an input component $u_{j}$ ) is called the output connectivity condition: 
Cond1. There exists an $\mathbf{Y}$-topped starting from $\mathbf{x}_{\mathbf{i}}$ (respectively $\mathbf{u}_{\mathbf{j}}$ ).

This condition is very easy to prove. In fact, assume that for some $x_{i}$, it is not satisfied. Then, we have that, $\forall k \geq 0$, the $i^{t h}$ column of $C^{\lambda}\left(A^{\lambda}\right)^{k}$ is equal to zero. So the output and all its derivatives are not sensitive to the state component $x_{i}$. Thus, $x_{i}$ is not observable. This reasoning can, obviously, be also done for input component $u_{j}$.

Since the output connectivity condition is only a necessary condition, the objective of the study below is to give the graphical necessary and sufficient conditions for the strong observability of a state or an input component. At this aim, we have recourse to the computation of the generic dimension of the strongly observable subspace in the extended state and input subspace $\left(x^{T}, u^{T}\right)^{T}$. As we will see below, this dimension is related to the function $\beta$.

Using the results of (Commault et al., 1997), where authors treat the disturbance rejection problem, we have that input components included in $\mathbf{U} \backslash \overline{\mathbf{U}}(\mathbf{Y})$ can be rendered unobservable using the $\overline{\mathbf{U}}(\mathbf{Y})$ components i.e. there exist inputs $\overline{\mathbf{U}}(\mathbf{Y})$ such that output $y(t)$ is not sensitive to the input components associated to $\mathbf{U} \backslash \overline{\mathbf{U}}(\mathbf{Y})$. Hence, the input components associated to vertices $\mathbf{U} \backslash \overline{\mathbf{U}}(\mathbf{Y})$ are not strongly observable and so, for a sake of simplicity, we restrict our observability study only to the input components associated to $\overline{\mathbf{U}}(\mathbf{Y})$.

Let us denote by $\bar{B}^{\lambda}$ (resp. $\bar{D}^{\lambda}$ ) the sub-matrix of $B^{\lambda}$ (resp. $D^{\lambda}$ ) associated to $\overline{\mathbf{U}}(\mathbf{Y})$ i.e. matrix $\bar{B}^{\lambda}$ (resp. $\bar{D}^{\lambda}$ ) is constituted by the concatenation of columns $B_{j}^{\lambda}\left(\right.$ resp. $D_{j}^{\lambda}$ ) of $B^{\lambda}$ (resp. $D^{\lambda}$ ) where $\mathbf{u}_{\mathbf{j}} \in \overline{\mathbf{U}}(\mathbf{Y})$. Let us denote $\bar{q}=\operatorname{card}(\overline{\mathbf{U}}(\mathbf{Y}))$ and the pencil matrix of system $\left(A^{\lambda}, \bar{B}^{\lambda}, C^{\lambda}, \bar{D}^{\lambda}\right)$ by $P^{\lambda}(s)=\left(\begin{array}{cc}A^{\lambda}-s I_{n} & \bar{B}^{\lambda} \\ C^{\lambda} & \bar{D}^{\lambda}\end{array}\right)$.

The system defined by matrices $\left(A^{\lambda}, \bar{B}^{\lambda}, C^{\lambda}, \bar{D}^{\lambda}\right)$ is generically input and state observable iff $g \_r a n k\left(P^{\lambda}(s)\right)=n+\bar{q}$ or in other words iff $P^{\lambda}(s)$ generically has full column rank. Otherwise, if all the state and input components are the start vertices of Y-topped paths and as $\rho[\overline{\mathbf{U}}(\mathbf{Y}), \mathbf{Y}]=\operatorname{card}(\overline{\mathbf{U}}(\mathbf{Y}))=\bar{q}$, using the results of (van der Woude, 2000), we have that the generic normal rank of $P^{\lambda}(s)$ is equal to $n+\bar{q}$. Thus, $g \_r a n k\left(P^{\lambda}\left(s_{0}\right)\right)<n+\bar{q}$ is equivalent (Trentelman et al., 2001) to the existence of a nonzero vector $\left(x_{0}^{T}, u_{0}^{T}\right)^{T}$ such that the output $y$ resulting from the initial conditions $u(t)=u_{0} e^{s_{0} t}$ and $x(0)=x_{0}$ is zero and so that there exists a 
direction in the extended state and input space which is not strongly observable. Consequently, the generic dimension of the strongly observable subspace in the extended state and input subspace $\left(x^{T}, u^{T}\right)^{T}$ is closely related to the generic number of invariant zeros of $P^{\lambda}(s)$ i.e. the complex roots of $g \_r a n k\left(P^{\lambda}(s)\right)<n+\bar{q}$ (Trentelman et al., 2001). Indeed, if we denote $g_{-} n_{i n v, z}$ this number, the generic dimension of the strongly observable subspace in the extended state and input subspace $\left(x^{T}, u^{T}\right)^{T}$ is equal to $n+\bar{q}-g \_n_{i n v, z}$.

Before giving the necessary and sufficient condition for the strong observability of a state or an input component, we give hereafter a graphical characterization of the generic dimension of the strongly observable subspace in the extended state and input subspace $\left(x^{T}, u^{T}\right)^{T}$. This characterization is based on the graphical computation of the number $g_{-} n_{i n v, z}$.

Lemma 1 Consider structured system $\Sigma_{\Lambda}$ represented by digraph $\mathcal{G}\left(\Sigma_{\Lambda}\right)$. We have that $n+\bar{q}-$ $g \_n_{i n v, z}=\beta(\mathbf{Y})$, where $g \_n_{i n v, z}$ is the number of invariant zeros of $P^{\lambda}(s)$.

Proof: Due to the properties of subdivision presented in Definition 3 (Boukhobza et al., 2007), we have that there is no edge from $\mathbf{X}_{\mathbf{0}}(\mathbf{Y}) \cup \mathbf{U}_{\mathbf{0}}(\mathbf{Y})$ to $\mathbf{X}_{\mathbf{1}}(\mathbf{Y}) \cup \mathbf{\Upsilon}_{\mathbf{1}}(\mathbf{Y})$ and $\mathbf{S}_{*}\left(\mathbf{U}_{\mathbf{0}}(\mathbf{Y}), \mathbf{Y}\right)=$ $\mathbf{X}_{\mathbf{S}}(\mathbf{Y}) \cup \Upsilon_{\mathbf{0}}(\mathbf{Y})$. Thus, we can write $\Sigma_{\Lambda}$ as:

$$
\left\{\begin{array}{l}
\dot{X}_{0}(t)=A_{0,0}^{\lambda} X_{0}(t)+A_{0, s}^{\lambda} X_{s}(t)+A_{0,1}^{\lambda} X_{1}(t)+B_{0,0}^{\lambda} U_{0}(t)+B_{0,1}^{\lambda} U_{1}(t) \\
\dot{X}_{s}(t)=A_{s, 0}^{\lambda} X_{0}(t)+A_{s, s}^{\lambda} X_{s}(t)+A_{s, 1}^{\lambda} X_{1}(t)+B_{s, 0}^{\lambda} U_{0}(t)+B_{s, 1}^{\lambda} U_{1}(t) \\
\dot{X}_{1}(t)=A_{1, s}^{\lambda} X_{s}(t)+A_{1,1}^{\lambda} X_{1}(t)+B_{1,1}^{\lambda} U_{1}(t) \\
\Upsilon_{0}(t)=C_{0,0}^{\lambda} X_{0}(t)+C_{0, s}^{\lambda} X_{s}(t)+C_{0,1}^{\lambda} X_{1}(t)+D_{0,0}^{\lambda} U_{0}(t)+D_{0,1}^{\lambda} U_{1}(t) \\
\Upsilon_{1}(t)=C_{1, s}^{\lambda} X_{s}(t)+C_{1,1}^{\lambda} X_{1}(t)+D_{1,1}^{\lambda} U_{0}(t)
\end{array}\right.
$$

where $X_{0}, X_{s}, U_{0}, U_{1}, \Upsilon_{0}$ and $\Upsilon_{1}$ represent the variables associated to vertex subsets $\mathbf{X}_{\mathbf{0}}(\mathbf{Y})$, $\mathrm{X}_{\mathbf{s}}(\mathbf{Y}), \mathbf{U}_{\mathbf{0}}(\mathbf{Y}), \mathbf{U}_{\mathbf{1}}(\mathbf{Y}), \Upsilon_{\mathbf{0}}(\mathbf{Y})$ and $\Upsilon_{\mathbf{1}}(\mathbf{Y})$ respectively.

Therefore, with some appropriate permutations on the rows and columns of $P^{\lambda}(s)$, we can transform $P^{\lambda}(s)$ into

$$
\tilde{P}^{\lambda}(s)=\left(\begin{array}{ccccc}
A_{0,0}^{\lambda}-s I_{n_{0}} & A_{0, s}^{\lambda} & B_{0,0}^{\lambda} & A_{0,1}^{\lambda} & B_{0,1}^{\lambda} \\
A_{s, 0}^{\lambda} & A_{s, s}^{\lambda}-s I_{n_{s}} & B_{s, 0}^{\lambda} & A_{s, 1}^{\lambda} & B_{s, 1}^{\lambda} \\
C_{0,0}^{\lambda} & C_{0, s}^{\lambda} & D_{0,0}^{\lambda} & C_{0,1}^{\lambda} & D_{0,1}^{\lambda} \\
0 & A_{1, s}^{\lambda} & 0 & A_{1,1}^{\lambda}-s I_{n_{1}} & B_{1,1}^{\lambda} \\
0 & C_{1, s}^{\lambda} & 0 & C_{1,1}^{\lambda} & D_{1,1}^{\lambda}
\end{array}\right)
$$


For a sake of simplicity, let us define $n_{0}=\operatorname{card}\left(\mathbf{X}_{\mathbf{0}}(\mathbf{Y})\right), n_{s}=\operatorname{card}\left(\mathbf{X}_{\mathbf{s}}(\mathbf{Y})\right), n_{1}=\operatorname{card}\left(\mathbf{X}_{\mathbf{1}}(\mathbf{Y})\right)$, $q_{0}=\operatorname{card}\left(\mathbf{U}_{\mathbf{0}}(\mathbf{Y})\right), q_{1}=\operatorname{card}\left(\mathbf{U}_{\mathbf{1}}(\mathbf{Y})\right), p_{0}=\operatorname{card}\left(\boldsymbol{\Upsilon}_{\mathbf{0}}(\mathbf{Y})\right)$ and $p_{1}=\operatorname{card}\left(\boldsymbol{\Upsilon}_{\mathbf{1}}(\mathbf{Y})\right)$.

Since the edges associated to $A_{1, s}^{\lambda} \operatorname{link} \mathbf{X}_{\mathbf{s}}(\mathbf{Y})$ to $\mathbf{X}_{\mathbf{1}}(\mathbf{Y})$ and the edges associated to $C_{1, s}^{\lambda}$ link $\mathbf{X}_{\mathbf{s}}(\mathbf{Y})$ to $\boldsymbol{\Upsilon}_{\mathbf{1}}(\mathbf{Y})$, we have that $g_{-} \operatorname{rank}\left(\begin{array}{c}A_{1, s}^{\lambda} \\ C_{1, s}^{\lambda}\end{array}\right)=\theta\left(\mathbf{X}_{\mathbf{s}}(\mathbf{Y}), \mathbf{X}_{\mathbf{1}}(\mathbf{Y}) \cup \boldsymbol{\Upsilon}_{\mathbf{1}}(\mathbf{Y})\right)$. According to Statement St3 of Lemma 6 in (Boukhobza et al., 2007), g_rank $\left(\begin{array}{c}A_{1, s}^{\lambda} \\ C_{1, s}^{\lambda}\end{array}\right)=n_{s}$ and so the number of invariant zeros of $P^{\lambda}(s)$ is equal to the number of invariant zeros of $P_{e}^{\lambda}(s)$, where

$$
P_{e}^{\lambda}(s)=\left(\begin{array}{cccccc}
A_{0,0}^{\lambda}-s I_{n_{0}} & A_{0, s}^{\lambda} & B_{0,0}^{\lambda} & A_{0,1}^{\lambda} & B_{0,1}^{\lambda} & 0 \\
A_{s, 0}^{\lambda} & A_{s, s}^{\lambda}-s I_{n_{s}} & B_{s, 0}^{\lambda} & A_{s, 1}^{\lambda} & B_{s, 1}^{\lambda} & 0 \\
C_{0,0}^{\lambda} & C_{0, s}^{\lambda} & D_{0,0}^{\lambda} & C_{0,1}^{\lambda} & D_{0,1}^{\lambda} & 0 \\
0 & I_{n_{s}} & 0 & 0 & 0 & 0 \\
0 & 0 & 0 & A_{1,1}^{\lambda}-s I_{n_{1}} & B_{1,1}^{\lambda} & A_{1, s}^{\lambda} \\
0 & 0 & 0 & C_{1,1}^{\lambda} & D_{1,1}^{\lambda} & C_{1, s}^{\lambda}
\end{array}\right)
$$

Let us denote $P_{0}^{\lambda}(s) \stackrel{\text { def }}{=}\left(\begin{array}{ccc}A_{0,0}^{\lambda}-s I_{n_{0}} & A_{0, s}^{\lambda} & B_{0,0}^{\lambda} \\ A_{s, 0}^{\lambda} & A_{s, s}^{\lambda}-s I_{n_{s}} & B_{s, 0}^{\lambda} \\ C_{0,0}^{\lambda} & C_{0, s}^{\lambda} & D_{0,0}^{\lambda} \\ 0 & I_{n_{s}} & 0\end{array}\right)$ and $P_{1}^{\lambda}(s) \stackrel{\text { def }}{=}$ $\left(\begin{array}{ccc}A_{1,1}^{\lambda}-s I_{n_{1}} & B_{1,1}^{\lambda} & A_{1, s}^{\lambda} \\ C_{1,1}^{\lambda} & D_{1,1}^{\lambda} & C_{1, s}^{\lambda}\end{array}\right)$.

Matrices $P_{0}^{\lambda}(s)$ and $P_{1}^{\lambda}(s)$ can be seen respectively as the pencil matrices of the square system denoted $\Sigma_{0}$ and the system denoted $\Sigma_{1}$, which has generically full column $n$-rank even after the deletion of an arbitrary row (Boukhobza et al., 2007).

From (van der Woude, 2000), g_n-rank $\left(P_{0}^{\lambda}(s)\right)$ is equal to the number of rows of $P_{0}^{\lambda}(s)$ and $g \_n$-rank $\left(P_{1}^{\lambda}(s)\right)$ is equal to the number of columns of $P_{1}^{\lambda}(s)$. Thus, counting the zeros with their multiplicities, the number of invariant zeros of $P_{e}^{\lambda}(s)$ is equal to the sum of the number of invariant zeros of $P_{0}^{\lambda}(s)$ and the number of invariant zeros of $P_{1}^{\lambda}(s)$. On the one hand, applying Theorem 5.1 of (van der Woude, 2000), we have that the number of invariant zeros of $P_{0}^{\lambda}(s)$ is equal to $n_{0}+n_{s}+q_{0}-\mu\left[\mathbf{U}_{\mathbf{0}}(\mathbf{Y}), \mathbf{S}_{*}\left(\mathbf{U}_{\mathbf{0}}(\mathbf{Y}), \mathbf{Y}\right)\right]+\rho\left[\mathbf{U}_{\mathbf{0}}(\mathbf{Y}), \mathbf{S}_{*}\left(\mathbf{U}_{\mathbf{0}}(\mathbf{Y}), \mathbf{Y}\right)\right]-n_{s}$. Note that the presence of the latter term $n_{s}$ is due to the fact that the output of system $\Sigma_{0}$ is $Y_{s}$ and not $X_{s}$. Moreover from Theorem 5.2 of (van der Woude, 2000), the number of invariant zeros of $P_{1}^{\lambda}(s)$ is equal to $n_{1}+n_{s}+q_{1}$ minus the maximal number of vertices of $\mathbf{X}_{\mathbf{1}}(\mathbf{Y}) \cup \mathbf{X}_{\mathbf{s}}(\mathbf{Y}) \cup \mathbf{U}_{\mathbf{1}}(\mathbf{Y})$ covered by a disjoint union of: 
- a $\mathbf{X}_{\mathbf{s}}(\mathbf{Y}) \cup \mathbf{U}_{\mathbf{1}}(\mathbf{Y})-\Upsilon_{\mathbf{1}}(\mathbf{Y})$ linking of size $\rho\left[\mathbf{X}_{\mathbf{s}}(\mathbf{Y}) \cup \mathbf{U}_{\mathbf{1}}(\mathbf{Y}), \boldsymbol{\Upsilon}_{\mathbf{1}}(\mathbf{Y})\right]$,

- a $\Upsilon_{1}(\mathbf{Y})$-topped path family and

- a cycle family covering only elements of $\mathbf{X}_{1}(\mathbf{Y})$.

Therefore, using notations of Definition 3, the number of invariant zeros of $P_{e}^{\lambda}(s)$ and also of $P^{\lambda}(s)$ is equal to $n_{0}+q_{0}+n_{1}+n_{s}+q_{1}-\beta_{0}(\mathbf{Y})-\beta_{1}(\mathbf{Y})=n+\bar{q}-\beta_{0}(\mathbf{Y})-\beta_{1}(\mathbf{Y})$. Thus, the generic dimension of the strongly observable subspace of $\Sigma_{\Lambda}$ in the extended state and input subspace is equal to $n+\bar{q}-g \_n_{i n v, z}=\beta_{1}(\mathbf{Y})+\mu\left[\mathbf{U}_{\mathbf{0}}(\mathbf{Y}), \mathbf{S}_{*}\left(\mathbf{U}_{\mathbf{0}}(\mathbf{Y}), \mathbf{Y}\right)\right]-\rho\left[\mathbf{U}_{\mathbf{0}}(\mathbf{Y}), \mathbf{S}_{*}\left(\mathbf{U}_{\mathbf{0}}(\mathbf{Y}), \mathbf{Y}\right)\right]=$ $\beta_{1}(\mathbf{Y})+\beta_{0}(\mathbf{Y})=\beta(\mathbf{Y})$.

The previous lemma allows us to write that the generic dimension of the strongly observable subspace in the extended state and input subspace $\left(x^{T}, u^{T}\right)^{T}$ is equal to $\beta(\mathbf{Y})$. The necessary and sufficient strong observability conditions can be deduced immediately from this Lemma. Indeed, if $\beta(\mathbf{Y})<n+q$ then $\Sigma_{\Lambda}$ is not generically input and state observable and it may be interesting to know which state component $x_{i}$ (resp. input component $u_{j}$ ) is generically strongly observable. At this aim, we compare $\beta\left(\mathbf{Y} \cup\left\{\mathbf{x}_{\mathbf{i}}\right\}\right)$ (resp. $\beta\left(\mathbf{Y} \cup\left\{\mathbf{u}_{\mathbf{j}}\right\}\right)$ ) to $\beta(\mathbf{Y})$. Indeed, this amounts to compare the generic dimension of the strongly observable subspace in the extended state and input subspace $\left(x^{T}, u^{T}\right)^{T}$ of $\Sigma_{\Lambda}$ to the generic dimension of the strongly observable subspace in the extended state and input subspace $\left(x^{T}, u^{T}\right)^{T}$ of the same system $\Sigma_{\Lambda}$ with an additional sensor which measures the component $x_{i}$ (resp. $u_{j}$ ). In fact, adding to the system a sensor, which measures the state component $x_{i}$ (resp. input component $u_{j}$ ) is equivalent to add in the digraph an output vertex $\mathbf{y}_{\mathbf{p}+\mathbf{1}}$ and an edge $\left(\mathbf{x}_{\mathbf{i}}, \mathbf{y}_{\mathbf{p}+\mathbf{1}}\right)$ (resp. $\left.\left(\mathbf{u}_{\mathbf{j}}, \mathbf{y}_{\mathbf{p}+\mathbf{1}}\right)\right)$. For the new system obtained by the addition of $\mathbf{y}_{\mathbf{p}+\mathbf{1}}$, the computation of the generic dimension of the strongly observable subspace in the extended state and input subspace $\left(x^{T}, u^{T}\right)^{T}$ can be made by using function $\beta\left(\mathbf{Y} \cup\left\{\mathbf{y}_{\mathbf{p}+\mathbf{1}}\right\}\right)$. Nevertheless, this requires an effective redraw of the digraph to add effectively an output vertex $\mathbf{y}_{\mathbf{p}+\mathbf{1}}$ and an edge $\left(\mathbf{x}_{\mathbf{i}}, \mathbf{y}_{\mathbf{p}+\mathbf{1}}\right)$ (resp. $\left.\left(\mathbf{u}_{\mathbf{j}}, \mathbf{y}_{\mathbf{p}+\mathbf{1}}\right)\right)$. For a sake of simplicity, we have chosen to work on an unique digraph. Thus, we do not add any vertex or edge in the digraph, but we consider vertex $\mathbf{x}_{\mathbf{i}}\left(\right.$ resp. $\mathbf{u}_{\mathbf{j}}$ ) as an output. Thus, it is easy to deduce from Lemma 1 that 
$\beta(\mathbf{V})=\beta_{1}(\mathbf{V})+\mu\left[\mathbf{U}_{\mathbf{0}}(\mathbf{V}), \mathbf{S}_{*}(\mathbf{V})\right]-\rho\left[\mathbf{U}_{\mathbf{0}}(\mathbf{V}), \mathbf{S}_{*}(\mathbf{V})\right]+\operatorname{card}(\mathbf{V} \backslash \mathbf{Y})$, for $\mathbf{V}=\mathbf{Y} \cup\left\{\mathbf{x}_{\mathbf{i}}\right\}$ (resp. $\mathbf{V}=\mathbf{Y} \cup\left\{\mathbf{u}_{\mathbf{j}}\right\}$ ), represents the generic dimension of the strongly observable subspace in the extended state and input subspace $\left(x^{T}, u^{T}\right)^{T}$ for the new system obtained by the addition of a measurement on $x_{i}$ (resp. $u_{j}$ ). Hence, we have:

Proposition 1 Consider structured system $\Sigma_{\Lambda}$ represented by digraph $\mathcal{G}\left(\Sigma_{\Lambda}\right)$. Let $\mathcal{V}_{\text {obs }} \stackrel{\text { def }}{=}$ $\{\mathbf{v} \in \mathbf{X} \cup \mathbf{U}, \beta(\mathbf{Y} \cup\{\mathbf{v}\})=\beta(\mathbf{Y})\}$. Assume that any state and input component is the start vertex of an $\mathrm{Y}$-topped path. State component $x_{i}$ (respectively input component $u_{j}$ ) is strongly observable iff

Cond1. Output connectivity condition: there exists an $\mathbf{Y}$-topped starting from $\mathbf{x}_{\mathbf{i}}\left(\right.$ res. $\left.\mathbf{u}_{\mathbf{j}}\right)$.

Cond2. $\beta$-condition $: \mathbf{x}_{\mathbf{i}} \in \mathcal{V}_{\text {obs }}\left(\right.$ resp. $\left.\mathbf{u}_{\mathbf{j}} \in \mathcal{V}_{\text {obs }}\right)$

Proof: Obviously, a state component $x_{i}$ is strongly observable iff an additional measure of this state component does not change the generic dimension of the strongly observable subspace. Using notations of Definition 3, this implies that state component $x_{i}$ (resp. input component $u_{j}$ ) is strongly observable iff $\beta(\mathbf{Y})=\beta\left(\mathbf{Y} \cup\left\{\mathbf{x}_{\mathbf{i}}\right\}\right)$ (resp. $\beta(\mathbf{Y})=\beta\left(\mathbf{Y} \cup\left\{\mathbf{u}_{\mathbf{j}}\right\}\right)$ ) and the proposition follows.

\subsection{Additional sensor for recovering the output connectivity condition}

Let us now come back to the sensor location problem and let us denote by $\Delta$ the vertex subset including all the vertices associated to the state and input components we want to observe. Similarly to the study (Commault et al., 2005), we subdivide our analysis into two parts. We first ensure that all the vertices of $\Delta$ are the start vertices of an $\mathrm{Y}$-topped path (output connectivity condition). When this condition is recovered for all the elements of $\Delta$, we treat the $\beta$-condition.

Let us denote by $\Delta_{1}$ the set of all the elements included $\Delta$ which are not the start vertex of an Y-topped path. The following definitions are similar to the ones given in (Commault et al., 2005). Two vertices $\mathbf{v}_{\mathbf{i}}$ and $\mathbf{v}_{\mathbf{j}}$ are said to be strongly connected if $\rho\left[\left\{\mathbf{v}_{\mathbf{i}}\right\},\left\{\mathbf{v}_{\mathbf{j}}\right\}\right]=\rho\left[\left\{\mathbf{v}_{\mathbf{j}}\right\},\left\{\mathbf{v}_{\mathbf{i}}\right\}\right]=1$. It is assumed that a vertex is connected to itself. The "strongly connected" relation noted $\mathcal{R}_{S C}$ 
is obviously an equivalence relation and we can define its equivalence classes. We denote each equivalent class by a strongly connected component of $\mathcal{G}\left(\Sigma_{\Lambda}\right)$. The strongly connected components can be ordered using a partial order relation “ß”defined as:

consider two strongly components $\mathbf{C}_{\mathbf{i}}$ and $\mathbf{C}_{\mathbf{j}}, \mathbf{C}_{\mathbf{i}} \preccurlyeq \mathbf{C}_{\mathbf{j}}$, if $\theta\left(\mathbf{C}_{\mathbf{j}}, \mathbf{C}_{\mathbf{i}}\right) \neq 0$.

Using relation “ß”, we can order partially all the strongly connected components. Let us denote by $I^{*}\left(\right.$ resp. $\left.J^{*}\right)$ the set of all components $\mathbf{x}_{\mathbf{i}} \in \Delta_{1}$ (resp. $\left.\mathbf{u}_{\mathbf{j}} \in \Delta_{1}\right)$ such that the strongly component including $\mathbf{x}_{\mathbf{i}}\left(\right.$ resp. $\left.\mathbf{u}_{\mathbf{j}}\right)$ is minimal in $\Delta_{1}$. For each element $\mathbf{x}_{\mathbf{i}} \in I^{*}$ and for each $\mathbf{u}_{\mathbf{j}} \in J^{*}$, we associate respectively $\Omega_{x, i}$ and $\Omega_{u, j}$ defined as $\Omega_{x, i}=\left\{\mathbf{x}_{\mathbf{k}} \in \mathbf{C}_{\ell}, \mathbf{C}_{\ell} \in \mathbf{X} / \mathcal{R}_{S C}\right.$ and $\left.\mathbf{C}_{\ell} \preccurlyeq \mathbf{C}_{\mathbf{x}_{\mathbf{i}}}\right\}$ and $\Omega_{u, j}=\left\{\mathbf{x}_{\mathbf{k}} \in \mathbf{C}_{\ell}, \mathbf{C}_{\ell} \in(\mathbf{X} \cup \mathbf{U}) / \mathcal{R}_{S C}\right.$ and $\left.\mathbf{C}_{\ell} \preccurlyeq \mathbf{C}_{\mathbf{x}_{\mathbf{i}}}\right\}$, where $\mathbf{X} / \mathcal{R}_{S C}\left(\operatorname{resp} .(\mathbf{X} \cup \mathbf{U}) / \mathcal{R}_{S C}\right)$ is the quotient de $\mathbf{X}$ (resp. $\mathbf{X} \cup \mathbf{U}$ ) by relation $\mathcal{R}_{S C}$. Using the previous statements, we have:

Proposition 2 Consider structured linear system $\left(\Sigma_{\Lambda}\right)$ represented by digraph $\mathcal{G}\left(\Sigma_{\Lambda}\right)$. To recover the output connectivity condition Cond1, the additional sensors must be sensitive at least to one state or input in each set $\Omega_{x, i}, \mathbf{x}_{\mathbf{i}} \in I^{*}$ and $\Omega_{u, j}, \mathbf{u}_{\mathbf{j}} \in J^{*}$.

Proof: The Proposition is easy to establish. We present hereafter only a sketch of its proof.

Sufficiency: On the one hand, by construction, all the state and input vertices included in $\Delta_{1}$ are connected to all the elements of at least one subset $\Omega_{x, i}, \mathbf{x}_{\mathbf{i}} \in I^{*}$ and $\Omega_{u, j}, \mathbf{u}_{\mathbf{j}} \in J^{*}$. Hence, if for each of these sets, one element is connected to an output then the output connectivity condition is satisfied.

Necessity: If there is a set $\Omega_{x, i}, \mathbf{x}_{\mathbf{i}} \in I^{*}$ or $\Omega_{u, j}, \mathbf{u}_{\mathbf{j}} \in J^{*}$ which has no vertex connected to an additional sensor, then there is no path between the corresponding vertex $\mathbf{x}_{\mathbf{i}} \in \Delta_{1}$ or $\mathbf{u}_{\mathbf{j}} \in \Delta_{1}$ and any of the measurements vertices. This implies that the output connectivity condition is not satisfied for this component even after the addition of new sensors and so elements of $\Delta_{1}$ cannot be strongly observable.

Proposition 2 indicates the location of the additional sensors but not their minimal number. Indeed, theoretically, it is possible that only one sensor, which, takes its measurements in each subset $\Omega_{x, i}, \mathbf{x}_{\mathbf{i}} \in I^{*}$ and $\Omega_{u, j}, \mathbf{u}_{\mathbf{j}} \in J^{*}$ (i.e. the measurement collected by the additional sensor is a 
linear combination of the state and input components which includes at least one element in every $\Omega_{x, i}, \mathbf{x}_{\mathbf{i}} \in I^{*}$ and $\Omega_{u, j}, \mathbf{u}_{\mathbf{j}} \in J^{*}$, allows to satisfy the requirements of the output connectivity condition. Nevertheless, this may be not practically feasible. Yet, it is obvious that adding more than $\operatorname{card}\left(I^{*} \cup J^{*}\right)$ sensors is useless to recover the output connectivity condition.

The sensor placement strategy which can de deduced from conditions of Proposition are illustrated in Subsection 4.4 with a double-effect pilot plant evaporator system.

\subsection{Additional sensor for recovering the $\beta$ condition}

The aim of the remaining part of this paper is to study additional sensor implementation in the case where the $\beta$-condition is not satisfied. For a sake of simplicity, we will proceed first by considering only the observability of one state or input component, and then we consider the set $\Delta$ associated to the state and input components to be observed.

From Lemma $1, \Delta_{2}=\Delta \backslash\{\mathbf{v} \in \Delta, \beta(\mathbf{Y} \cup\{\mathbf{v}\})=\beta(\mathbf{Y})\}$ represents all the components of $\Delta$ which are not strongly observable. For each component $\mathbf{x}_{\mathbf{i}} \in \Delta_{2}\left(\right.$ resp. $\left.\mathbf{u}_{\mathbf{j}} \in \Delta_{2}\right)$, we define $\Gamma_{x, i}=\left\{\mathbf{v} \in \mathbf{X} \cup \mathbf{U}, \beta\left(\mathbf{Y} \cup\left\{\mathbf{v}, \mathbf{x}_{\mathbf{i}}\right\}\right)=\beta(\mathbf{Y} \cup\{\mathbf{v}\})\right\}\left(\right.$ resp. $\Gamma_{u, j}=\{\mathbf{v} \in \mathbf{X} \cup \mathbf{U}, \beta(\mathbf{Y} \cup$ $\left.\left.\left.\left\{\mathbf{v}, \mathbf{u}_{\mathbf{i}}\right\}\right)=\beta(\mathbf{Y} \cup\{\mathbf{v}\})\right\}\right)$. We can enounce the following Lemma:

Lemma 2 Consider structured linear system $\left(\Sigma_{\Lambda}\right)$ represented by digraph $\mathcal{G}\left(\Sigma_{\Lambda}\right)$. For each component $\mathbf{x}_{\mathbf{i}} \in \Delta_{2}$ (resp. $\mathbf{u}_{\mathbf{j}} \in \Delta_{2}$ ), to recover the $\beta$ condition, it is sufficient to add a sensor which takes all its measurements in $\Gamma_{x, i} \cup \mathcal{V}_{\text {obs }}$ (resp. $\Gamma_{u, j} \cup \mathcal{V}_{o b s}$ ) with exactly one measurement in $\Gamma_{x, i}$ (resp. in $\Gamma_{u, j}$ ) or in other words an additional sensor $z$ which is a linear combination of components in $\mathcal{V}_{\text {obs }}$ and a component in $\Gamma_{x, i}\left(\right.$ resp. in $\left.\Gamma_{u, j}\right)$.

Proof: The proof of this Lemma is immediate knowing that the minimal number of sensors to recover the observability of $x_{i}$ (resp. $u_{j}$ ) is equal to one. Since $\mathcal{V}_{o b s}$ represents the strongly observable components, a sensor which measures a linear combination of components in $\mathcal{V}_{\text {obs }}$ and a component in $\Gamma_{x, i}$ (resp. in $\Gamma_{u, j}$ ) is equivalent to a sensor which measures only an element of $\Gamma_{x, i}$ (resp. in $\Gamma_{u, j}$ ). Moreover, from the previous settings, considering a vertex $\mathbf{v}$, 
the equality $\beta\left(\mathbf{Y} \cup\left\{\mathbf{v}, \mathbf{x}_{\mathbf{i}}\right\}\right)=\beta(\mathbf{Y} \cup\{\mathbf{v}\})$ is equivalent to say that a sensor which measures the component associated to $\mathbf{v}$ guarantees the strong observability of $x_{i}$ (resp. $u_{j}$ when $\beta\left(\mathbf{Y} \cup\left\{\mathbf{v}, \mathbf{u}_{\mathbf{j}}\right\}\right)=\beta(\mathbf{Y} \cup\{\mathbf{v}\})$.

Let us consider now all the components of set $\Delta_{2}$ and let us order subsets $\Gamma_{x, i}$ and (resp. $\Gamma_{u, j}$ ) according to the usual inclusion relation " $\subseteq$ ". Let $\Delta_{2}^{*}$ denotes the subset of $\Delta_{2}$ such that $\Delta_{2}^{*} \stackrel{\text { def }}{=}\left\{\mathbf{x}_{\mathbf{i}} \in \Delta_{\mathbf{2}}, \Gamma_{x, i}\right.$ is a minimal element w.r.t. relation" $\subseteq$ " $\} \cup\left\{\mathbf{u}_{\mathbf{j}} \in\right.$ $\Delta_{2}, \Gamma_{\mathbf{u}, \mathbf{j}}$ is a minimal element w.r.t. relation" $\subseteq$ " $\}$. In the sequel, we define a new output vector $z$ associated to the additional sensors collecting the new measurements $z(t)=H_{x}^{\lambda}(t) x+H_{u}^{\lambda} u(t)$. The additional sensor components can be represented by vertex set $\mathbf{Z}$ and edge subsets $H_{x}$-edges and $H_{u}$-edges from respectively $\mathbf{X}$ to $\mathbf{Z}$ and $\mathbf{U}$ to $\mathbf{Z}$. These edges reflect the location of the additional sensors. The following proposition gives sufficient conditions on the additional sensors to ensure the strong observability of all the elements included in $\Delta$.

Proposition 3 Consider structured linear system $\left(\Sigma_{\Lambda}\right)$ represented by digraph $\mathcal{G}\left(\Sigma_{\Lambda}\right)$. Assume that each element of $\Delta$ is the start vertex of an $\mathrm{Y}$-topped path. To recover the strong observability of the state and input components associated to the elements of $\Delta$ with additional sensors represented by vertex set $\mathbf{Z}$, it is sufficient to have:

$$
\begin{aligned}
& \exists \mathbf{V}_{\mathbf{X}, \mathbf{U}} \subseteq\left(\bigcup_{x_{i} \in \Delta_{2}^{*}} \boldsymbol{\Gamma}_{\mathbf{x}, \mathbf{i}}\right) \cup\left(\bigcup_{u_{j} \in \Delta_{2}^{*}} \boldsymbol{\Gamma}_{\mathbf{u}, \mathbf{j}}\right), \text { such that } \\
& \left\{\begin{array}{l}
\forall \mathbf{x}_{\mathbf{i}} \in \Delta_{2}^{*}, \mathbf{V}_{\mathbf{X}, \mathbf{U}} \cap \boldsymbol{\Gamma}_{\mathbf{x}, \mathbf{i}} \neq \emptyset \\
\forall \mathbf{u}_{\mathbf{j}} \in \Delta_{2}^{*}, \mathbf{V}_{\mathbf{X}, \mathbf{U}} \cap \boldsymbol{\Gamma}_{\mathbf{u}, \mathbf{j}} \neq \emptyset \\
\theta\left((\mathbf{X} \cup \mathbf{U}) \backslash \mathcal{V}_{o b s}, \mathbf{Z}\right)=\operatorname{card}\left(\mathbf{V}_{\mathbf{X}, \mathbf{U}}\right)+\theta\left((\mathbf{X} \cup \mathbf{U}) \backslash\left(\mathcal{V}_{o b s} \cup \mathbf{V}_{\mathbf{X}, \mathbf{U}}\right), \mathbf{Z}\right) \\
\theta\left(\mathbf{V}_{\mathbf{X}, \mathbf{U}}, \mathbf{Z}\right)=\operatorname{card}\left(\mathbf{V}_{\mathbf{X}, \mathbf{U}}\right)
\end{array}\right.
\end{aligned}
$$

Proof: System equation (4) ensures that for each $\mathbf{x}_{\mathbf{i}} \in \Delta_{2}^{*}\left(\right.$ resp. $\mathbf{u}_{\mathbf{j}} \in \Delta_{2}^{*}$ ) there exists an element in $\Gamma_{\mathbf{x}, \mathbf{i}}\left(\right.$ resp. $\Gamma_{\mathbf{u}, \mathbf{j}}$ ) which is a linear combination of the added sensors $z$ and elements of $\mathcal{V}_{\text {obs }}$ and which is then strongly observable. Moreover, since $\Gamma_{x, i}, \mathbf{x}_{\mathbf{i}} \in \Delta_{2}^{*}$ and $\Gamma_{u, j}, \mathbf{u}_{\mathbf{j}} \in \Delta_{2}^{*}$ are minimal w.r.t inclusion relation, then $\forall \mathbf{x}_{\mathbf{k}} \in \Delta_{2}$ (resp. $\forall \mathbf{u}_{\ell} \in \Delta_{2}$ ), there exists $\mathbf{x}_{\mathbf{i}} \in \Delta_{2}^{*}$ or $\mathbf{u}_{\mathbf{j}} \in \Delta_{2}^{*}$ such that $\boldsymbol{\Gamma}_{\mathbf{x}, \mathbf{k}} \subseteq \boldsymbol{\Gamma}_{\mathbf{x}, \mathbf{i}}\left(\right.$ resp. $\left.\boldsymbol{\Gamma}_{\mathbf{u}, \ell} \subseteq \boldsymbol{\Gamma}_{\mathbf{x}, \mathbf{i}}\right)$ or $\boldsymbol{\Gamma}_{\mathbf{x}, \mathbf{k}} \subseteq \boldsymbol{\Gamma}_{\mathbf{u}, \mathbf{j}}\left(\right.$ resp. $\left.\boldsymbol{\Gamma}_{\mathbf{u}, \ell} \subseteq \boldsymbol{\Gamma}_{\mathbf{u}, \mathbf{j}}\right)$. Hence, for each $\mathbf{x}_{\mathbf{i}} \in \Delta_{2}$ 
(resp. $\mathbf{u}_{\mathbf{j}} \in \Delta_{2}$ ), there exists an element in $\boldsymbol{\Gamma}_{\mathbf{x}, \mathbf{i}}\left(\right.$ resp. $\boldsymbol{\Gamma}_{\mathbf{u}, \mathbf{j}}$ ) which is a linear combination of the added sensors $z$ and elements of $\mathcal{V}_{o b s}$. According to Lemma 2, this is sufficient to ensure the strong observability of all the elements of $\Delta_{2}$.

To illustrate the previous result, consider system described in Example 1. On the one hand, we have that $\mathcal{V}_{o b s}=\left\{\mathbf{u}_{\mathbf{2}}, \mathbf{x}_{\mathbf{5}}, \mathbf{x}_{\mathbf{8}}, \mathbf{x}_{\mathbf{9}}\right\}$. On the other hand $\Gamma_{x, 1}=\left\{\mathbf{x}_{\mathbf{1}}\right\}, \Gamma_{x, 2}=\left\{\mathbf{x}_{\mathbf{2}}\right\}$, $\Gamma_{x, 3}=\left\{\mathbf{x}_{\mathbf{3}}, \mathbf{x}_{\mathbf{4}}, \mathbf{x}_{\mathbf{6}}\right\}, \Gamma_{x, 4}=\Gamma_{x, 6}=\left\{\mathbf{x}_{\mathbf{4}}, \mathbf{x}_{\mathbf{6}}\right\}, \Gamma_{x, 7}=\Gamma_{x, 10}=\left\{\mathbf{x}_{\mathbf{7}}, \mathbf{x}_{\mathbf{1 0}}\right\}$ and $\Gamma_{u, 1}=$ $\left\{\mathbf{u}_{\mathbf{1}}\right\}$. The minimal elements w.r.t. inclusion relation are $\Gamma_{x, 1}, \Gamma_{x, 2}, \Gamma_{x, 4}, \Gamma_{x, 6}, \Gamma_{x, 7}, \Gamma_{x, 10}$ and $\Gamma_{u, 1}$. Assume that we want to observe elements of $\Delta=\left\{\mathbf{u}_{1}, \mathbf{x}_{\mathbf{3}}, \mathbf{x}_{\mathbf{4}}, \mathbf{x}_{\mathbf{7}}, \mathbf{x}_{\mathbf{1 0}}\right\}$. Here $\Delta_{2}=\Delta$ and $\Delta_{2}^{*}=\left\{\mathbf{u}_{\mathbf{1}}, \mathbf{x}_{\mathbf{4}}, \mathbf{x}_{\mathbf{7}}, \mathbf{x}_{10}\right\}$. Since, $\Gamma_{x, 7}=\Gamma_{x, 10}$, we have that 3 sensors are sufficient if they satisfy constraints of system (4). For example, the additional measurements $z_{1}=\alpha_{1,1} x_{4}+\alpha_{1,2} x_{7}+\phi_{1}\left(u_{2}, x_{5}, x_{8}, x_{9}\right), z_{2}=\alpha_{2,1} x_{7}+\alpha_{2,2} u_{1}+\phi_{2}\left(u_{2}, x_{5}, x_{8}, x_{9}\right), z_{3}=$ $\alpha_{3,1} x_{4}+\alpha_{3,2} x_{7}+\alpha_{3,3} u_{1}+\phi_{3}\left(u_{2}, x_{5}, x_{8}, x_{9}\right)$ make the components associated to $\Delta$ generically observable.

Note that Proposition 3 does not give necessary conditions to be satisfied by the additional sensors except the obvious one $\beta(\mathbf{Y} \cup \mathbf{Z} \cup \boldsymbol{\Delta})=\beta(\mathbf{Y} \cup \mathbf{Z})$. Furthermore, it does not provide any information on the minimal number of additional sensors necessary to ensure the strong observability of $\Delta$. Indeed, we can just guarantee that there exists a solution with $p^{*}$ additional sensors where $p^{*}$ is $\min \left(\operatorname{card}\left(\mathbf{V}_{\mathbf{X}, \mathbf{U}}\right)\right)$ under the constraints $\forall \mathbf{x}_{\mathbf{i}} \in \Delta_{2}^{*}, \mathbf{V}_{\mathbf{X}, \mathbf{U}} \cap \boldsymbol{\Gamma}_{\mathbf{x}, \mathbf{i}} \neq \emptyset$ and $\forall \mathbf{u}_{\mathbf{j}} \in \Delta_{2}^{*}, \mathbf{V}_{\mathbf{X}, \mathbf{U}} \cap \boldsymbol{\Gamma}_{\mathbf{u}, \mathbf{j}} \neq \emptyset$.

\subsection{Example}

In this example, the proposed results are applied to place sensors in order to ensure the observability of some state and input components of a double-effect pilot plant evaporator represented by a fifth-order linear state-space model (Buchholt and Kümmel, 1981; Phatak and Viswanadham, 1988; Xiong and Saif, 2003). The system's functioning is described in (Buchholt and Kümmel, 1981; Phatak and Viswanadham, 1988) and can be summarized as follows : The feed solution is pumped into a first effect. The first effect solution is heated by saturated steam and the boil-off 
travels into the second effect steam jacket. The concentrated solution from the first effect enters the second effect which operates under vacuum. The concentrated product is pumped to storage. More detailed description of the evaporator is presented in (Buchholt and Kümmel, 1981).

The fifth-order model uses the state variables $x_{1}$ which is first-effect holdup, the first effect concentration $x_{2}$, the first-effect temperature $x_{3}$, the second effect holdup $x_{4}$ and the second effect concentration $x_{5}$. The control known variables are not taken into account. In addition to the state and control variables there are also unknown inputs inputs to the process $u_{1}$ which represents the variations in feed flow rate and $u_{2}$ associated to the variations in feed concentration.

The digraph representation of the considered linearized model is given in Figure 2.

Assume that we want to ensure the observability of the state components $x_{1}, x_{2}, x_{4}$ and $x_{5}$ and

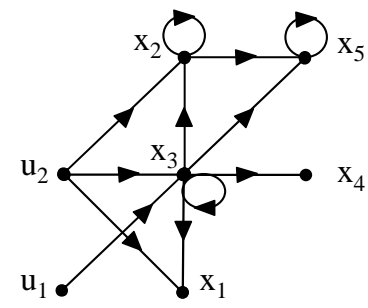

Figure 2: Digraph representation of a double-effect pilot plant evaporator

also the input component $u_{1}$ i.e. $\Delta=\left\{\mathbf{x}_{1}, \mathbf{x}_{2}, \mathbf{x}_{4}, \mathbf{x}_{\mathbf{5}}, \mathbf{u}_{\mathbf{1}}\right\}$. Note that $\Delta_{1}=\Delta$ since we consider that initially there is no measurement on the system as during a conception stage. First we interest in recovering the output connectivity condition. Each state or input vertex constitutes a strongly connected components i.e. $\left\{\mathbf{u}_{1}\right\},\left\{\mathbf{u}_{2}\right\},\left\{\mathbf{x}_{1}\right\},\left\{\mathbf{x}_{2}\right\},\left\{\mathbf{x}_{3}\right\},\left\{\mathbf{x}_{4}\right\}$ and $\left\{\mathbf{x}_{5}\right\}$. Moreover, we have the following relations $\left\{\mathbf{x}_{5}\right\} \preceq\left\{\mathbf{x}_{2}\right\} \preceq\left\{\mathbf{x}_{3}\right\} \preceq\left\{\mathbf{u}_{1}\right\},\left\{\mathbf{x}_{4}\right\} \preceq\left\{\mathbf{x}_{3}\right\} \preceq\left\{\mathbf{u}_{2}\right\}$ and $\left\{\mathbf{x}_{1}\right\} \preceq\left\{\mathbf{x}_{3}\right\}$. The minimal elements in $\Delta$ are $\left\{\mathbf{x}_{1}\right\},\left\{\mathbf{x}_{4}\right\}$ and $\left\{\mathbf{x}_{5}\right\}$. Thus, $I^{*}=\left\{\mathbf{x}_{1}, \mathbf{x}_{4}, \mathbf{x}_{5}\right\}$ and $J^{*}=\emptyset$. This leads to the following sets $\Omega_{x, 1}=\left\{\mathbf{x}_{1}\right\}, \Omega_{x, 4}=\left\{\mathbf{x}_{4}\right\}$ and $\Omega_{x, 5}=\left\{\mathbf{x}_{\mathbf{5}}\right\}$.

Therefore, to satisfy the output connectivity condition, we must have sensors which are sensitive to $x_{1}, x_{4}$ and $x_{5}$. Note that only one sensor is sufficient if it measures a linear combination of $x_{1}$, $x_{4}$ and $x_{5}$ i.e. $z=\alpha_{1} x_{1}+\alpha_{4} x_{4}+\alpha_{5} x_{5}+\phi\left(x_{1}, x_{2}, x_{3}, u_{1}, u_{2}\right)$, where $\phi$ is any linear function. Assume that we add two sensors $z_{1}=\alpha_{1} x_{1}$ and $z_{2}=\alpha_{4} x_{4}+\alpha_{5} x_{5}$ to satisfy the output con- 
nectivity condition. Let us now focus on the $\beta$-condition. We have to compute $\boldsymbol{\Gamma}_{\mathbf{x}, \mathbf{1}}, \boldsymbol{\Gamma}_{\mathbf{x}, \mathbf{2}}$, $\Gamma_{\mathbf{x}, 4}, \Gamma_{\mathbf{x}, 5}$ and $\boldsymbol{\Gamma}_{\mathbf{u}, \mathbf{1}}$. Relations of Definition 3 allow us to write that $\beta\left(\left\{\mathbf{x}_{\mathbf{1}}\right\}\right)=5$ while $\beta\left(\left\{\mathbf{x}_{\mathbf{2}}\right\}\right)=\beta\left(\left\{\mathbf{x}_{\mathbf{3}}\right\}\right)=\beta\left(\left\{\mathbf{x}_{\mathbf{4}}\right\}\right)=\beta\left(\left\{\mathbf{x}_{\mathbf{5}}\right\}\right)=\beta\left(\left\{\mathbf{u}_{\mathbf{1}}\right\}\right)=\beta\left(\left\{\mathbf{u}_{\mathbf{2}}\right\}\right)=7$. Furthermore, $\mathcal{V}_{\text {obs }}=\left\{\mathbf{x}_{1}\right\}$. Thus, we can deduce that $\Gamma_{\mathbf{x}, \mathbf{2}}=\Gamma_{\mathbf{x}, 4}=\Gamma_{\mathbf{x}, 5}=\Gamma_{\mathbf{u}, 1}=\left\{\mathbf{x}_{2}, \mathbf{x}_{\mathbf{3}}, \mathbf{x}_{\mathbf{4}}, \mathbf{x}_{\mathbf{5}}, \mathbf{u}_{\mathbf{1}}, \mathbf{u}_{\mathbf{2}}\right\}$. Thus, only one additional sensor is sufficient to ensure the strong observability of elements associated to $\Delta$ if it is sensitive to any of the state components $x_{2}, x_{3}, x_{4}, x_{5}$ or to the input components $u_{1}$ and $u_{2}$. Thus, the complete solution necessitates three sensors $z_{1}=\alpha_{1} x_{1}, z_{2}=\alpha_{4} x_{4}+\alpha_{5} x_{5}$ and $z_{3}$ which can take any measurement on the input or on the state components under the constraint that $z_{1}, z_{2}$ and $z_{3}$ are linearly independent. Note that for this example, it is not possible to find a solution with less than three sensors.

\section{Concluding remarks}

In this paper, we propose, on the basis of a graph-theoretic approach, an analysis tool to study the number and the location of additional sensors in order to recover generic input and state observability of structured linear systems. First, we provide the graphical necessary and sufficient conditions which ensure the strong observability of any state or input component. Then, we study, the problem of additional sensors implementation which allows to recover these conditions.

Since we treat systems with unknown inputs, the approach based on bipartite graphs developed in (Commault et al., 2005) is not adapted. Indeed, output connectivity condition and $\theta(\mathbf{X} \cup \mathbf{U}, \mathbf{X} \cup \mathbf{Y})=n+q$ are not sufficient to ensure the observability of structured system $\Sigma_{\Lambda}$. A new function $\beta$ is then used to characterize the generic dimension of the strong observability subspace of the considered system. Otherwise, this function can also reflect the efficiency of a given sensor location.

Our approach uses classical programming techniques and is free from numerical difficulties since it uses well-known combinatorial techniques. This makes our proposed approach well suited to large scale systems. 
Further works addressing the problem of finding the exhaustive list, as well as the problems of finding the minimal number and the better sensors locations (w.r.t. to some criterion) which allow to recover the strong observability of any given part of the state and the input vectors.

\section{References}

G. Basile and G. Marro, "A new characterization of some structural properties of linear systems: unknowninput observability, invertibility and functional controllability," International Journal of Control, vol. 17, no. 5, pp. 931-943, 1973.

T. Boukhobza, F. Hamelin, and S. Martinez-Martinez, "State and input observability for structured linear systems: a graph-theoretic approach,” Automatica, vol. 43, no. 7, pp. 1204-1210, 2007.

F. Buchholt and M. Kümmel, "A multivariable selftuning regulator to control a double effect evaporator," Automatica, vol. 17, no. 5, pp. 737-743, 1981.

D. Chu, "Disturbance decoupled observer design for linear time-invariant systems: a matrix pencil approach," IEEE Transactions on Automatic Control, vol. 45, no. 8, pp. 1569-1575, 2000.

D. Chu and V. Mehrmann, "Disturbance decoupled observer design for descriptor systems," System \& Control Letters, vol. 38, no. 1, pp. 37-48, 1999.

C. Commault, J. M. Dion, and V. Hovelaque, "A geometric approach for structured systems: application to disturbance decoupling," Automatica, vol. 33, no. 3, pp. 403-409, 1997.

C. Commault, J.-M. Dion, and D. H. Trinh, "Observability recovering by additional sensor implementation in linear structured systems," in Proceedings of the $44^{\text {th }}$ IEEE Conference on Decision and Control, and the European Control Conference, Seville, Spain, 2005.

M. Darouach, "Reduced-order observers for linear neutral delay systems," IEEE Transactions on Automatic Control, vol. 50, no. 9, pp. 1407-1413, 2005.

J.-M. Dion, C. Commault, and J. W. van der Woude, "Generic properties and control of linear structured systems: a survey," Automatica, vol. 39, no. 7, pp. 1125-1144, 2003.

H. J. Gao and C. H. Wang, "Delay-dependent Robust $H_{\infty}$ and $L_{2}-L_{\infty}$ filtering for a class of uncertain nonlinear time-delay systems," IEEE Transactions on Automatic Control, vol. 48, no. 9, pp. 1661-1666, 2003.

M. L. J. Hautus, "Strong detectability and observers," Linear Algebra and its Applications, vol. 50, pp. 353-360, 1983.

M. Hou and P. C. Müller, "Design of observers for linear systems with unknown input," IEEE Transactions on Automatic Control, vol. 37, no. 4, pp. 871-875, 1992.

M. Hou and P. C. Müller, "Causal observability of descriptor systems," IEEE Transactions on Automatic Control, vol. 44, no. 1, pp. 158-163, 1999.

M. Hou and R. J. Patton, "Input observability and input reconstruction," Automatica, vol. 34, no. 6, pp. 789-794, 1998.

M. Hou, A. C. Pugh, and P. C. Müller, "Disturbance decoupled functional observer," IEEE Transactions on Automatic Control, vol. 44, no. 2, pp. 382-386, 1999. 
D. Koenig, "Unknown input proportional multiple-integral observer design for linear descriptor systems: application to state and fault estimation," IEEE Transactions on Automatic Control, vol. 50, no. 2, pp. 212-217, 2005.

P. Kudva, N. Viswanadham, and A. A. Ramakrishna, "Observers for linear systems with unknown inputs," IEEE Transactions on Automatic Control, vol. 25, no. 2, pp. 113-115, 1980.

C. Lin, Q. G. Wang, T. H. Lee and Y. He, "Fuzzy weighting-dependent approach to $h_{\infty}$ filter design for time-delay fuzzy systems," IEEE Transactions on Signal Processing, vol. 55, no. 6, Part 1, pp. 27462751, 2007.

D. Maquin, M. Luong, and J. Ragot, "Observability analysis and sensor placement," in IFAC SAFEPROCESS'94/IMACS Symposium on Fault Detection, Supervision and Safety for Technical Process, Espoo, Finland, 1994.

M. Meyer, J. M. L. Lann, B. Koehret, and M. Enjalbert, "Optimal selection of sensor location on a complex plant using a graph oriented approach," Computers \& Chemical Engineering, vol. 18, pp. S535-S540, 1994.

M. S. Phatak and N. Viswanadham, "Actuator Fault Detection and Isolation in Linear Systems," International Journal of Systems Science, vol. 19, no. 12, pp. 2593-2603, 1988.

H. L. Trentelman, A. A. Stoorvogel, and M. Hautus, Control Theory for Linear Systems. London, U.K. Springer, 2001.

H. Trinh and Q. Ha, "Design of linear functional observers for linear systems with unknown inputs," International Journal of Systems Science, vol. 31, no. 6, pp. 741-749, 2000.

C. C. Tsui, "A new design approach to unknown input observers," IEEE Transactions on Automatic Control, vol. 41, no. 3, pp. 464-468, 1996.

M. van de Wal and B. de Jager, "A review of methods for input/output selection," Automatica, vol. 37, no. 4, pp. 487-510, 2001.

J. W. van der Woude, "The generic number of invariant zeros of a structured linear system," SIAM Journal of Control and Optimization, vol. 38, no. 1, pp. 1-21, 2000.

J. W. van der Woude, C. Commault, and J. M. Dion, "Zero orders and dimensions of some invariant subspaces in linear structured systems," Mathematics of Control, Signals and Systems, vol. 16, pp. 225237, 2003.

Y. Xiong and M. Saif, "Unknown Disturbance Inputs Based on a State Functional Observer Design," Automatica, vol. 39, no. 8, pp. 1389-1398, 2003. 